\title{
Actinomycetic Corneal Ulcer
}

\author{
MOHINDER SINGH and BALJIT KAUR
}

Kuala Lumpur

\begin{abstract}
Summary
A rare case of keratoactinomycosis developing in the absence of any known ocular trauma is described. It showed a dramatic response to penicillin therapy. Steroids should be cautiously used in the presence of active corneal disease. This case highlights the importance of repeated examination of corneal scrapings.
\end{abstract}

Actinomycosis is a rare cause of ulcerative keratitis. Early identification of the organism is essential for effective therapy. The infection normally occurs following trivial corneal injury especially when contaminated by vegetable matter and it is therefore more common in outdoor labourers. We report a case where keratoactinomycosis developed in the absence of any known ocular trauma and its definitive diagnosis was delayed.

\section{Case Report}

G.S. a 28 years old male field health worker engaged in collecting mosquitoes presented at a peripheral eye clinic on June 1, 1984 with a one day history of redness of his left eye. He was treated with 'some eye drops'. Three days later he noticed a white spot in the lower part of the 'black' of his left eye. He was started on gutt. chloromycetin two hourly, gutt. atropine daily, gutt. sofradex two hourly and oc. cambisone at night. A depot steroid was injected subconjunctivally. He was also given $60 \mathrm{mg}$ oral predinisolone each day. However, his symptoms got worse and he was referred to our clinic on June 7, 1984. When we first saw him he complained of progressive visual haze, increasing ocular pain and photophobia along with constant watering of his left eye.

Right visual acuity was $6 / 6$ and left $6 / 12$ corrected. The left eye was markedly congested and showed folliculopapillary response in the inferior conjunctiva. A whitish mass of depot steroid was noticed in the lower fornix. The left cornea showed an irregular superficial ulcer about $5 \times 3 \mathrm{~mm}$ mainly confined to the lower temporal quadrant. Discrete grey-white satellite stromal infiltrates were present adjacent to the advancing upper edge of the ulcer. The ulcer bed was relatively clean and rather dry looking. The limbal tissue near the ulcerated area was conspicuously inflamed. (Fig. 1) The anterior chamber had moderate activity but no hypopyon. Ocular tension felt normal digitally and the posterior segment was unremarkable.

Systemic examination was noncontributary. There was no history of any ocular trauma or local ophthalmic medication. He was not using contact lenses.

A gram smear on the first visit revealed a few pus cells but no organisms. A 48 hour culture was negative.

He was started on gutt. gentamycin hourly along with gutt. atropine twice daily. Oral indomethacin $25 \mathrm{mg}$ twice daily was also prescribed which was increased to $50 \mathrm{mg}$ twice daily two days later. One gram of vitamin $\mathrm{C}$ was also given daily. The corneal lesion did not show any improvement. Hourly instillation of gutt. amphoteracin was added one week later and the subconjunctival depot steroid was excised. However, the pain persisted, the eye became more congested and corneal ulceration spread further into the central zone. Blood vessels started to invade the cornea from below and a hypopyon appeared by the third week after admission. The left visual acuity dropped to 6/60. (Fig. 2)

A fresh gram smear was prepared from the 


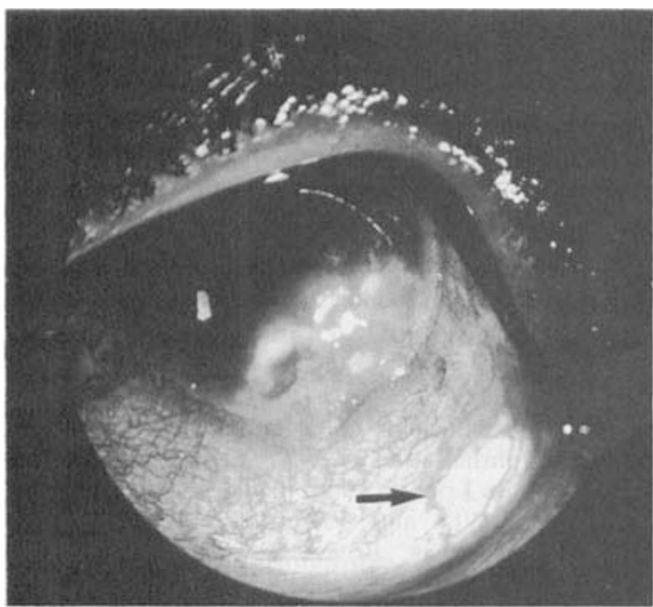

Fig. 1. Whitish depot steroid (arrow) and corneal ulceration.

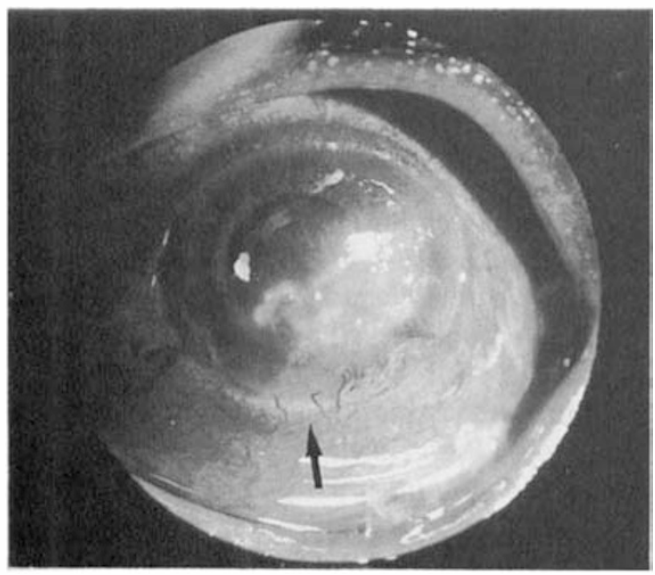

Fig. 2. Progression of corneal ulcer with hypopyon (arrow) and vascular invasion.

advancing edge of the ulcer. It revealed the presence of typical gram positive Actinomyces (Fig. 3). Half hourly topical penicillin $(200,000 \mathrm{u} / \mathrm{ml})$ therapy together with a daily dose of 1.2 mega units of parenteral penicillin was immediately commenced. The previous medication was stopped. The corneal ulcer began to improve within 72 hours and complete healing occurred within ten days. The vision improved to $6 / 18$. Penicillin therapy was completed and stopped after two weeks.

The corneal blood vessels regressed and a superficial corneal scar was left. When reviewed on January 21, 1986 the left vision had improved to $6 / 12$ though a faint corneal opacity persisted. (Fig. 4)

\section{Discussion}

Two genera of the family of Actinomycetacae produce ulcerative lesions of the cornea. Anaerobic Actinomyces is a relatively more common cause of keratoactinomycosis than aerobic Nocardia. ${ }^{1}$ Only careful culture techniques can identify the specific organism. Most reported cases have occurred due to Actinomyce israeli ${ }^{1}$ but one case due to Actinomyce bovis has been described. ${ }^{2}$ The clinical progress is usually slow and torpid but occasionally can become rapidly progressive even in the form of a ring abscess. ${ }^{1}$ The deep seated form of keratoactinomycosis rarely becomes deep enough to perforate the cornea and cause loss of the eye. ${ }^{3}$ This observation is also supported by our case in whom the ulceration remained limited to the anterior one third of the stromal thickness throughout

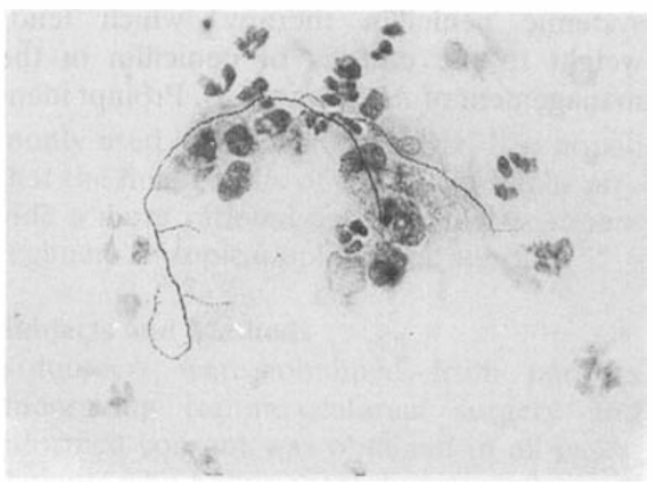

Fig. 3. Photomicrograph of corneal scraping showing typical actinomyces (x 1000)

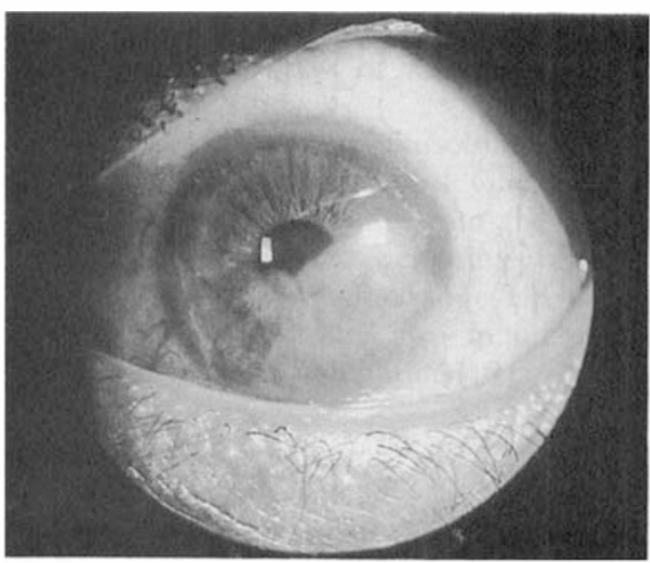

Fig. 4. Healed corneal ulcer. 
its violent course inspite of late recognition of its true aetiology.

A primary Actinomycetic corneal ulcer always follows an injury. ${ }^{1}$ It is not at all surprising because the unabraded corneal surface remains well oxygenated and is therefore not conducive to invasion by anaerobic organisms like Actinomyces. We were unable to elicit any evidence of ocular trauma in our case although occurence of a trivial corneal abrasion cannot be ruled out as many such injuries pass unnoticed.

It has been emphasised by many authors that application of steroids to the eye favours the onset of fungal infection. ${ }^{4.5}$ This might have happened in our patient by the liberal use of steroids. The clinician should be extremely careful in the use of steroids in the presence of an active corneal ulcer. Our case showed a dramatic response to topical and systemic penicillin therapy, which lends weight to the efficacy of penicillin in the management of Actinomycosis. Prompt iden- tification of the organism is therefore necessary to initiate appropriate treatment. Gram smears and cultures must be done repeatedly in any case of recalcitrant corneal ulcer especially if it fails to respond to conventional therapy.

The authors are grateful to Puan Husna Idris for her secretarial help and Encik Alias Manap for the photographic work.

\section{References}

${ }^{1}$ Duke-Elder S: Inflammations of the Cornea. System of Ophthalmology. Vol. VIII part 2 Henry Kimpton London 1965. p. 788-90.

${ }^{2}$ Gingrich WD and Pinkerton ME: Anaerobic Actinomycosis Bovis Corneal Ulcer. Arch Ophthalmol 1962, 67: 549-53.

${ }^{3}$ François $\mathbf{J}$ and Rysselaere $\mathrm{M}$ : Actinomycotic infections. Oculomycosis. Charles C. Thomas, Springficld Illinois 1972. p. 135-36.

${ }^{4}$ Mitsui $\mathrm{Y}$ and Hanabusa J: Corneal infections after cortisone therapy. Br J Ophthalmol 1955, 39: 24450.

${ }^{5}$ Ley AP and Sanders TE: Fungus Keratitis. A report of Three cases. Arch Ophthalmol 1956, 56: 25764. 